\title{
Redox interactions of nitric oxide with dopamine and its derivatives
}

\author{
Fernando Antunes $^{\mathrm{a}, *}$, Carla Nunes $^{\mathrm{b}}$, João Laranjinha $^{\mathrm{b}}$, Enrique Cadenas $^{\mathrm{c}}$ \\ a Instituto de Investigação Científica Bento da Rocha Cabral and Grupo de Bioquimica dos Oxidantes e Antioxidantes, \\ Centro de Quimica e Bioquimica, Universidade de Lisboa, Portugal \\ ${ }^{\mathrm{b}}$ Faculty of Pharmacy and Center for Neuroscience and Cell Biology, University of Coimbra, Portugal \\ ${ }^{\mathrm{c}}$ Department of Molecular Pharmacology and Toxicology, University Southern California, Los Angeles, CA, USA
}

Available online 4 January 2005

\begin{abstract}
Nitric oxide $(\bullet \mathrm{NO})$ is a ubiquitous diffusible messenger in the central nervous system. ${ }^{\bullet} \mathrm{NO}$ and derived nitrogen species may interact with catecholamines, thus, modifying not only its regulatory actions but also producing oxidants and free radicals that are likely to trigger toxic pathways in the nervous system. Oxidative pathways and chain oxidation reactions triggered by catecholamines may be broken by ascorbate and glutathione, of which there is ample supply in the brain. At the subcellular level, mitochondria and cytosolic dopamine storage vesicles are likely to provide site-specific settings for ${ }^{\bullet} \mathrm{NO}$ and catecholamines interactions. Thus, a complex picture emerges in which the steady- state levels of the individual reactants, the rate constants of the reactions involved, the oxygen tension, and the compartmentalization of reactions determine the biological significance of the redox interactions between ${ }^{\bullet} \mathrm{NO}$ and dopamine metabolism in the brain. The physiological relevance of ${ }^{\bullet} \mathrm{NO}$-driven chemical modifications of dopamine and its derivatives and the ensuing free radical production are discussed in connection with the neurodegeneration inherent in Parkinson's disease.
\end{abstract}

(C) 2004 Elsevier Ireland Ltd. All rights reserved.

Keywords: Parkinson's disease; DOPAC; Mitochondria; Storage vesicles; Oxidative stress; Free radicals

\section{Introduction}

Parkinson's disease is one of the most prevalent age-associated neurodegenerative disorders characterized by the degeneration of neuromelanin-pigmented dopaminergic neurons in the pars compacta of the substantia nigra. Although the mechanisms underlying

\footnotetext{
* Corresponding author.

E-mail address: fantunes@fc.ul.pt (F. Antunes).
}

this disease are unknown, efforts towards the identification of the fundamental causes of Parkinsons's disease are based on the recognition of unique features of dopaminergic cells (Ebadi and Sharma, 2003; Mandel et al., 2003): (1) the restriction of cell death to the dopamine-producing neurons has focused much attention on the dopamine molecule itself as an important factor; (2) mitochondrial dysfunction and oxidative stress appear to be two major contributors to degeneration of dopaminegic neurons; (3) ${ }^{\bullet} \mathrm{NO}$ increases 
during the progress of Parkinson's diseases as a result of inflammation-like processes and fosters dopamine depletion; the ensuing neurotoxicity is averted by nNOS inhibitors; and (4) ${ }^{\bullet} \mathrm{NO}$ participates in the etiology of the disease as indicated by the protective effect against 1-methyl-4-phenyl-1,2,3,6-tetrahydropyridine (MPTP)-induced dopaminergic degeneration observed both in a mutant mice lacking the inducible nitric oxide synthase (iNOS) (Klivenyi et al., 2000) and in a mutant mice lacking the nNOS gene.

Despite the involvement of ${ }^{\bullet} \mathrm{NO}$ in catecholamine metabolism, the chemical interaction of dopamine and its metabolites with ${ }^{\bullet} \mathrm{NO}$ constitutes a source of neurotoxic molecules, which may contribute in the cellular process leading to neurodegeneration. In this work, we review some recent data on the redox interaction between ${ }^{\bullet} \mathrm{NO}$ and dopamine and its derivatives as well as the implications for the mitochondrial damage and neurodegeneration associated with Parkinsons's disease caused by these interactions.

\subsection{Redox chemistry of $N O$ with dopamine and derivatives}

-NO does not react directly with dopamine in anaerobic conditions (Laranjinha and Cadenas, 2002; Rettori et al., 2002), but in the presence of $\mathrm{O}_{2}$ dopamine is readily oxidized. The species thought responsible for this reaction is the product of the trimolecular reaction of $\mathrm{O}_{2}$ with ${ }^{\bullet} \mathrm{NO}$ (reaction (1)), ${ }^{\bullet} \mathrm{NO}_{2}$, or at high $\bullet^{\bullet} \mathrm{NO}$ concentrations (in the $\mathrm{mM}$ range) $\mathrm{N}_{2} \mathrm{O}_{3}$ (dinitrogen trioxide), which is the product of the reaction between ${ }^{\bullet} \mathrm{NO}_{2}$ with -NO (reaction (2)). Concerning the products formed, $\mathrm{N}_{2} \mathrm{O}_{3}$ — being a strong nitrosating agent-leads to nitrosation with the ensuing 6-nitrosodopamine formation (reaction (3)), which slowly is converted to 6-nitrodopamine, whereas ${ }^{\bullet} \mathrm{NO}_{2}$ leads to the formation of the semiquinone radical (reaction (4)).

$$
\begin{aligned}
& 2 \bullet \mathrm{NO}+\mathrm{O}_{2} \rightarrow 2 \bullet \mathrm{NO}_{2} \\
& \bullet \mathrm{NO}+{ }^{\bullet} \mathrm{NO}_{2} \rightarrow \mathrm{N}_{2} \mathrm{O}_{3}
\end{aligned}
$$

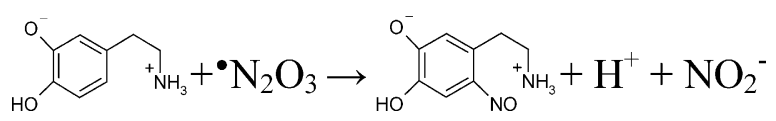

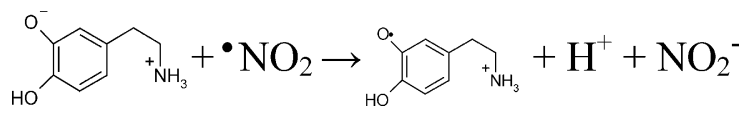

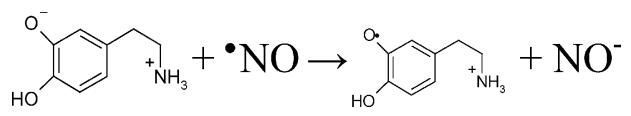

Concurring with dopamine oxidation, 3,4dihydroxyphenylacetic acid (DOPAC), a major metabolite of dopamine resulting from two oxidative steps catalysed by monoamine oxidase and aldehyde dehydrogenase in mitochondria, and 6-hydroxydopamine, a sympathetic neurotoxin, originated from the oxidation of dopamine, for very low oxygen tensions undergo one-electron oxidation by -NO forming the semiquinone and the nitroxyl anion $\left(\mathrm{NO}^{-}\right)$(reaction (5)).

At the low ${ }^{-N O}$ concentrations found in biological systems electron abstraction is expected to be the main dopamine oxidation pathway. The semiquinone formed can undergo either an oxidative or a reductive decay. Reaction with oxygen yields the quinone and the superoxide anion $\left(\mathrm{O}_{2}{ }^{--}\right)$(reaction (6)); the latter can continue the oxidative chain by reacting with ${ }^{\bullet} \mathrm{NO}$, at diffusion-controlled rates $\left(k=1.9 \times 10^{10} \mathrm{M}^{-1} \mathrm{~s}^{-1}\right)$ and resulting in peroxynitrite $\left(\mathrm{ONOO}^{-}\right)$formation (reaction (7)). Protonation of $\mathrm{ONOO}^{-}$to peroxynitrous acid $(\mathrm{ONOOH})$ involves a cage reaction (reaction (8)) containing both $\mathrm{HO}^{\bullet}$ and ${ }^{\bullet} \mathrm{NO}_{2}$, which can promote the one-electron oxidation of dopamine or any of its derivatives to the semiquinone (reaction (9)). Alternatively, the one-electron reduction of the semiquinone species to the quinol can be accomplished by cell reductants, such as ascorbate or glutathione (reaction (10)) (Laranjinha and Cadenas, 2002). If the local concentration of the semiquinone species is high, disproportionation can occur (reaction (11)).

$$
\begin{aligned}
& \bullet \mathrm{NO}+\mathrm{O}_{2}^{\bullet-} \rightarrow \mathrm{ONOO}^{-} \\
& \mathrm{ONOO}^{-}+\mathrm{H}^{+} \rightarrow \mathrm{ONOOH} \leftrightarrow\left[\mathrm{HO}^{\bullet} \ldots \mathrm{NO}_{2}^{\bullet}\right]
\end{aligned}
$$




$$
\text { (or } \mathrm{Asc}^{\bullet} \text { ) }
$$

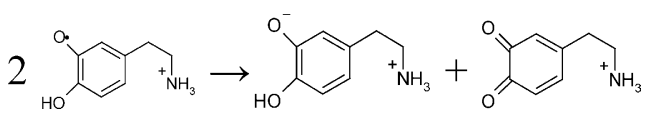

Besides the formation of dopamine oxidation products, which by themselves can be harmful for the cell (see below), the interaction between ${ }^{\bullet} \mathrm{NO}$ and dopamine can be an important source of oxidants, such as $\mathrm{O}_{2}{ }^{\bullet-}$ and $\mathrm{ONOO}-$, or lead to the oxidation of antioxidants, such as glutathione or ascorbate. Overall, these can constitute an important source of oxidative stress in the cell.

Taking in account that at the low ${ }^{\bullet} \mathrm{NO}$ concentration found in vivo the trimolecular reaction of ${ }^{\bullet} \mathrm{NO}$ with $\mathrm{O}_{2}$ is slow, the ${ }^{\bullet} \mathrm{NO}$-derived oxidant that is expected to be more relevant for the oxidation of dopamine in vivo is $\mathrm{ONOO}^{-}$. However, for those dopamine-derived species that can be directly oxidized by ${ }^{\bullet} \mathrm{NO}$, this reaction is potentially relevant, for it can evade the control by antioxidant systems, such as superoxide dismutase (SOD). In fact, it has been shown that SOD, by removing $\mathrm{O}_{2}{ }^{\bullet-}$, protects dopamine from oxidation by ${ }^{\bullet} \mathrm{NO}$ and inhibits the formation of $\mathrm{ONOO}^{-}$under aerobic conditions (Riobó et al., 2002). Interestingly, $\mathrm{Cu}, \mathrm{Zn}-\mathrm{SOD}$ enhances semiquinone formation in anaerobiosis by a mechanism that may involve oxidation of $\mathrm{NO}^{-}$back to ${ }^{\bullet} \mathrm{NO}$, hence increasing semiquinone formation (Laranjinha and Cadenas, 2002). Thus, under such conditions $\mathrm{Cu}, \mathrm{Zn}$-SOD imposes a kinetic control of $\bullet \mathrm{NO}$-mediated DOPAC oxidation accomplished within a cycle involving the ${ }^{\bullet} \mathrm{NO} \rightarrow \mathrm{NO}^{-}$transition during DOPAC oxidation (reaction (5)) and the $\mathrm{NO}^{-} \rightarrow{ }^{\bullet} \mathrm{NO}$ transition coupled to the reduction of $\mathrm{Cu}^{2+}$ in superoxide dismutase.

\subsection{Biological relevance of NO and dopamine chemical interactions}

Dopamine damaging effects to the cell can be envisaged by the following general pathways: (a) the production of $\mathrm{H}_{2} \mathrm{O}_{2}$ during the oxidative deamination of dopamine catalysed by monamine oxidase (MAO) present in the outer mitochondrial membrane; (b) the electrophilic character of quinones derived from dopamine oxidation that can directly damage several cellular components including the mitochondrion; and (c) dopamine chain oxidation that generates reactive oxygen species in a self-propagated cycle driven by $\mathrm{O}_{2}{ }^{\bullet-}$.

Concerning the first pathway, the levels of MAO in dopaminergic neurons are controversial, and probably only low amounts of MAO are present in dopaminergic cells (Hida et al., 1999). Moreover, independently of the levels of MAO in dopaminergic neurons, if the cytosolic dopamine concentration is taken into account $(\approx 1 \mu \mathrm{M})$ the reaction catalysed by MAO, which has a $K_{\mathrm{M}} \approx 650 \mu \mathrm{M}$ (Hauptmann et al., 1996), will be severely limited by the low dopamine concentration. It can, thus, be estimated that the production of $\mathrm{H}_{2} \mathrm{O}_{2}$ by this pathway is not significant when compared with other cellular sources of $\mathrm{H}_{2} \mathrm{O}_{2}$, such as mitochondrial respiration (Antunes et al., 2002).

The potential harmful biological actions of electrophilic species, such as quinones, is well documented (Brunmark and Cadenas, 1989), but the mechanisms of dopamine oxidation leading to electrophilic quinones as well as their quantitative importance are difficult to access. Concerning the initial step of dopamine oxidation either $\mathrm{O}_{2}{ }^{\bullet-}$ or $\mathrm{ONOO}^{-}$are likely candidates. Species like the hydroxyl radical or ${ }^{\bullet} \mathrm{NO}_{2}$ may not be formed in high enough amounts in order to be quantitatively important. A criterion to distinguish among these species on their relative importance for dopamine oxidation is the intracellular localization of dopamine in dopaminergic cells. The final steps of dopamine synthesis take place in the cytosol; once synthesized dopamine is rapidly transported via an ATP-dependent process into storage vesicles (Flatmark, 2000). An important characteristic of these vesicles is their acidic $\mathrm{pH}$ (approximately 5.5) which is thought to protect dopamine from oxidation, since the anion form of dopamine is the oxidizable form, while the protonated form is stabilized to oxidation. Nevertheless, due to the 
much higher concentration of dopamine in the storage vesicles $(>1 \mathrm{mM})$ compared with that in the cytosol (Brautigam et al., 1985), the concentration of the anion form of dopamine in the vesicles is expected to be approximately seventeen-fold higher than in the cytosol, in spite of the low $\mathrm{pH}$ in the vesicles (Antunes et al., 2002).

Thus, the oxidation of dopamine should be analyzed not only in the cytosol but as well in the storage vesicles. In the vesicles, the formation of superoxide is expect to be slow, and its migration from the cytosol to these vesicles is not probable to be significant because superoxide does not cross biomembranes, and so all superoxide that could cross the membrane would be in the form of the hydroperoxyl (Gus'kova et al., 1984), its protonated form. On the other hand, there is evidence of physical association between storage vesicles and mitochondria through tubular channels (Carmichael and Smith, 1978), and for the mitochondrial origin of the storage vesicles (Issidorides et al., 1996). So, the possibility of superoxide diffusing directly from mitochondria, one of the main cellular sites concerning superoxide production, to the vesicles should not be discarded. Concerning $\bullet \mathrm{NO}$, it can cross biomembranes freely (Subczynski et al., 1996), implying that the membrane of the vesicles does not provide protection against NO formed extracellularly or intracellularly. Once inside these vesicles, ${ }^{\bullet} \mathrm{NO}$ could react with the low amounts of superoxide and form $\mathrm{ONOO}^{-}$, which could initiate the oxidation of dopamine. Subsequently, this process could proceed in a self-propagated cycle formed by reactions (6)-(9). In the storage vesicles, dopamine oxidation could be expected to be a significant local source of superoxide radicals. Independently, of the precise mechanism there is now evidence of dopamine oxidation occurring in the storage vesicles, including processes mediated by ${ }^{\bullet} \mathrm{NO}$ (Antunes et al., 2002).

Concerning the cytosol, $\mathrm{O}_{2}{ }^{\bullet-}$ can be present in this site at high levels, namely through the leakage of superoxide from the respiratory chain in a process controlled by VDAC (Han et al., 2003). Therefore, the direct initiation of dopamine oxidation by $\mathrm{O}_{2}{ }^{\bullet-}$ is a plausible scenario. This oxidation could be increased in the presence of ${ }^{\bullet} \mathrm{NO}$ via two pathways: (a) increase of superoxide production caused by ${ }^{\bullet} \mathrm{NO}$ acting on the respiratory chain in an antimycin-like effect (Poderoso et al., 1996; (b) ${ }^{\bullet} \mathrm{NO}$ interaction with superoxide forming $\mathrm{ONOO}^{-}$, and thus, initiating dopamine oxidation. Dopamine ox- idation in the cytosol can assume some relevance if the storage is impaired leading to increased cytosolic levels (Lotharius and Brundin, 2002).

In addition to dopamine oxidation, nitration by ${ }^{\bullet} \mathrm{NO}$ -derived nitrogen oxides (most likely $\mathrm{N}_{2} \mathrm{O}_{3}$ ) has been reported to occur in vitro when ${ }^{\circ} \mathrm{NO}$ gas was bubbled into an aerobic solution of the catecholamine (Daveu et al., 1997). However, the high ${ }^{\bullet} \mathrm{NO}$ concentration (up to $\mathrm{mM}$ ) required turns questionable its physiological relevance even under inflammatory conditions (i.e., following expression of inducible iNOS in microglia and astrocytes) in which ${ }^{\bullet} \mathrm{NO}$ concentration is supposed to increase.

Associated with dopamine oxidation there are welldefined cellular effects that eventually could lead to dopaminergic cell death. A central theme in this regard is mitochondrial damage. Dopamine quinones can directly damage the mitochondrion by impairing the respiratory chain among other effects (Berman and Hastings, 1999; Ben-Shachar et al., 1995). Moreover, dopamine can potentiate the ${ }^{\bullet} \mathrm{NO}$-damaging effect on the respiratory chain (Antunes et al., 2002), causing apoptotic death in neurons by an early insult to mitochondria (Wallace et al., 1997; Berman and Hastings, 1999). An impaired mitochondrial respiration will cause a decrease ATP production, which is absolutely necessary for the storage of dopamine in the vesicles. This may cause an increase in the cytosolic levels of dopamine, where dopamine oxidation is more feasible than in the storage vesicles (Lotharius and Brundin, 2002), further increasing the mitochondrial damage. In this context, it is also relevant to note that dopamine quinones directly impair the uptake of dopamine into the storage vesicles (Terland et al., 1997). So, independent of the precise molecular mechanism involved, the basis for a self-propagated cycle which amplifies the initial damage are set, leading ultimately to the death of the dopaminergic cell. It may also be added that DOPAC potentiates ${ }^{\bullet} \mathrm{NO}$-induced cell death in PC12 cells by mechanisms that are likely to involve impairment of mitochondrial respiration (unpublished observations). This observation is in connection with the notion that mitochondria provides a sitespecific setting for the oxidation of DOPAC by ${ }^{\bullet} \mathrm{NO}$ (Laranjinha and Cadenas, 2002): First, DOPAC synthesis is expected to occur in the mitochondrial matrix via the activity of unspecific aldehyde dehydrogenase (Ambroziak and Pietruszko, 1991; Tank et al., 1981); 
second, the steady-state level of ${ }^{\bullet} \mathrm{NO}$ and $\mathrm{O}_{2}{ }^{\bullet-}$, in the mitochondrial matrix is expected to be high (Boveris et al., 2000), contributing to peroxynitrite formation (reaction (7)). Under these circumstances, the oxidation of DOPAC by $\mathrm{ONOO}^{-}$may be an important source of DOPAC semiquinone and ensuing redox transitions of this species.

Overall, it may be surmised that the redox chemistry described here involving, on the one hand, ${ }^{\bullet} \mathrm{NO}$ and derived nitrogen species and, on the other hand, dopamine and DOPAC, may be a source of signals that positively reinforce cellular pathways leading to cell death associated with Parkinson's disease. Nevertheless, one should also mention that glutathione and ascorbate, two antioxidants abundant in the brain in the $\mathrm{mM}$ range, are able to reduce $o$-semiquinones (Laranjinha and Cadenas, 2002) to the parent phenolic compounds (reaction (10)). Thus, by favouring the reductive decay of $o$-semiquinones, GSH and ascorbate prevent the formation of superoxide radical (reaction (6)) and the subsequent production of toxic species (reactions (7)-(9)). Finally, compartmentalization of reactions, either in vesicles or mitochondria, provides site-specific settings that facilitate oxidation of catecholamines and ensuing production of free radicals. A complex picture emerges where the steady-state levels of the individual reactants, the rate constants of the reactions involved, the oxygen tension and the compartmentalization of reactions will set the boundaries for dopamine and its metabolites as potential targets for ${ }^{\bullet} \mathrm{NO}$ and derived oxidants in the brain, affecting the physiological chemistry of both, •NO and catecholamines.

\section{Acknowledgements}

Supported by grants from Fundação Ciência e Tecnologia and FEDER (POCTI/2001/BCI/42365 and POCTI/2001/BCI/42245) and grant 5R01ES011342 from NIH. FA acknowledges financial support from FCT - Portugal (BPD/11487/2002).

\section{References}

Ambroziak, W., Pietruszko, R., 1991. Human aldehyde dehydrogenase. Activity with aldehyde metabolites of monoamines, diamines, and polyamines. J. Biol. Chem. 266, 13011-13018.
Antunes, F., Han, D., Rettori, D., Cadenas, E., 2002. Mitochondrial damage by nitric oxide is potentiated by dopamine in PC12 cells. Biochim. Biophys. Acta 1556, 233-238.

Ben-Shachar, D., Zuk, R., Glinka, Y., 1995. Dopamine neurotoxicity: inhibition of mitochondrial respiration. J. Neurochem. 64, 718-723.

Berman, S.B., Hastings, T.G., 1999. Dopamine oxidation alters mitochondrial respiration and induces permeability transition in brain mitochondria: implications for Parkinson's disease. J. Neurochem. 73, 1127-1137.

Boveris, A., Costa, L.E., Poderoso, J.J., Carreras, M.C., Cadenas, E., 2000. Regulation of mitochondrial respiration by oxygen and nitric oxide. Ann. N. Y. Acad. Sci. 899, 121-135.

Brautigam, M., Kittner, B., Herken, H., 1985. Evaluation of neurotropic drug actions on tyrosine hydroxylase activity and dopamine metabolism in clonal cell lines. Arzneimittelforschung. 35, 277-284.

Brunmark, A., Cadenas, E., 1989. Redox and addition chemistry of quinoid compounds and its biological implications. Free Radic. Biol. Med. 7, 435-477.

Carmichael, S.W., Smith, D.J., 1978. Direct connections between mitochondria and catecholamine-storage vesicles demonstrated by high voltage electron microscopy in rat adrenal medulla. Cell Tissue Res. 191, 421-432.

Daveu, C., Servy, C., Dendane, M., Marin, P., Ducrocq, C., 1997. Oxidation and nitration of catecholamines by nitrogen oxides derived from nitric oxide. Nitric Oxide 1, 234-243.

Ebadi, M., Sharma, S.K., 2003. Peroxynitrite and mitochondrial dysfunction in the pathogenesis of Parkinson's disease. Antioxid. Redox Signal. 5, 319-335.

Flatmark, T., 2000. Catecholamine biosynthesis and physiological regulation in neuroendocrine cells. Acta Physiol. Scand. 168, $1-17$.

Gus'kova, R.A., Ivanov, I.I., Akhobadze, V.V., Rubin, A.R., 1984. Permeability of bilayer lipid membranes for superoxide $\left(\mathrm{O}_{2}{ }^{\bullet-}\right)$ radicals. Biochim. Biophys. Acta 778, 579-583.

Han, D., Antunes, F., Canali, R., Rettori, D., Cadenas, E., 2003. Voltage-dependent anion channels control the release of the superoxide anion from mitochondria to cytosol. J. Biol. Chem. 278, 5557-5563.

Hauptmann, N., Grimsby, J., Shih, J.C., Cadenas, E., 1996. The metabolism of tyramine by monoamine oxidase A/B causes oxidative damage to mitochondrial DNA. Arch. Biochem. Biophys. 335, 295-304.

Hida, T., Hasegawa, Y., Arai, R., 1999. Histochemical study of dopamine-degrading monoamine oxidase activity in dopaminergic neurons of rat brain. Brain Res. 842, 491-495.

Issidorides, M.R., Kriho, V., Pappas, G.D., 1996. The fine structure of large dense-core organelles in human locus coeruleus neurons. Neurol. Res. 18, 57-63.

Klivenyi, P., Andreassen, O.A., Ferrante, R.J., Lancelot, E., Reif, D., Beal, M.F., 2000. Inhibition of neuronal nitric oxide synthase protects against MPTP toxicity. Neuroreport. 11, 12651268.

Laranjinha, J., Cadenas, E., 2002. Oxidation of DOPAC by nitric oxide. Effect of superoxide dismutase. J. Neurochem. 81, 892900. 
Lotharius, J., Brundin, P., 2002. Pathogenesis of Parkinson's disease: dopamine, vesicles and alpha-synuclein. Nat. Rev. Neurosci. 3, 932-942.

Mandel, S., Grunblatt, E., Riederer, P., Gerlach, M., Levites, Y., Youdim, M.B., 2003. Neuroprotective strategies in Parkinson's disease: an update on progress. CNS Drugs 17 (10), 729-762.

Poderoso, J.J., Carreras, M.C., Lisdero, C., Riobo, N., Schopfer, F., Boveris, A., 1996. Nitric oxide inhibits electron transfer and increases superoxide radical production in rat heart mitochondria and submitochondrial particles. Arch. Biochem. Biophys. 328, 85-92.

Rettori, D., Tang, Y., Dias Jr., L.C., Cadenas, E., 2002. Pathways of dopamine oxidation mediated by nitric oxide. Free Radic. Biol. Med. 33, 685-690.

Riobó, N.A., Schöpfer, F.J., Boveris, A.D., Cadenas, E., Poderoso, J.J., 2002. The reaction of nitric oxide with 6-hydroxydopamine: implications for Parkinson's disease. Free Radic. Biol. Med. 32, $115-121$.
Subczynski, W.K., Lomnicka, M., Hyde, J.S., 1996. Permeability of nitric oxide through lipid bilayer membranes. Free Radic. Res. 24, 343-349.

Tank, A.W., Weiner, H., Thurman, J.A., 1981. Enzymology and subcellular localization of aldehyde oxidation in rat liver. Oxidation of 3,4-dihydroxyphenylacetaldehyde derived fromdopamine to 3,4-dihydroxyphenylacetic acid. Biochem. Pharmacol. 30, 3265-3275.

Terland, O., Flatmark, T., Tangeras, A., Gronberg, M., 1997. Dopamine oxidation generates an oxidative stress mediated by dopamine semiquinone and unrelated to reactive oxygen species. J. Mol. Cell. Cardiol. 29, 1731-1738.

Wallace, D.C., Lott, M.T., Brown, M.D., 1997. Mitochondrial defects in neurodegenartive diseases and aging. In: Beal, M.F., Howel, N., Bodis-Wollner, I. (Eds.), Mitochondria and Free Radicals in Neurodegenerative Diseases. Wiley-Liss, Inc., New York, pp. 277-289. 